\title{
On Global and Nonlinear Symmetries in Quantum Mechanics
}

\author{
H.-D. Doebner
}

\section{Prolog}

I first met Jiri Niederle around 40 years ago in the International Centre for Theoretical Physics at Trieste. During this time the Centre was located in a modern building at Piazza Oberdan, the Director was Abdus Salam and his deputy was Paolo Budini. Jiri and I were fellows in a group under the indirect guidance of Asim Barut and Chris Fronsdal. I remember some our colleagues in this group: Arno Bohm, Richard Raczka, Moshe Flato. It was the period in which Abdus asked the scientist in the centre 'to push forward the frontiers of knowledge', especially in particle physics through models based on group theory; $\widetilde{U(12)}$ was fashionable in these times.

Some of us expected a description of the geometrical structure behind fundamental quantum physics through groups, their Lie algebras and their representations to be too narrow and not flexible enough to model real physical classical and quantum systems: If the group and their linear representation are chosen, there is not enough freedom to accommodate their characteristic properties. Hence, we were interested to apply a framework which is beyond groups. We tried e.g. nonlinear and nonintegrable Lie-algebra representations, nonseparable Hilbert spaces and Lie algebras over unusual number fields; we studied differential geometric methods which reflect local as well as global geometrical, algebraic, analytic and symmetry properties which are more general compared with group theory.

Jiri Niederle and Jouko Mickelsson were interested in the construction of nonlinear representations, which opens the plethora of unknown possibilities to model Lie symmetries of quantum systems. These representations contain nonlinear generators acting in a linear representation space. One has to specify the type of 'nonlinearity' and their physical interpretation.

Jiri Tolar and I myself intended to model quantum systems localized and moving on a smooth manifold $M$ with (classical) position and momentum observable $\mathfrak{O}$ which span their kinematics $K(M)$ through a quantization $Q(M, K)$ of $K(M)$ - BOREL kinematics - and, after the introduction of a time $t$ dependence, their dynamical properties - BOREL quantization - through e.g. as quantum analogous to the classical case.

It is reasonable to connect a paper on the occasion of the 70th birthday of Jiri Niederle with a retrospec- tive view on both of our attempts. We thought in Trieste that these ideas - the utilization of nonlinear representations and the application of global methods were not directly related to each other. However, it turns out now that this is not the case.

A discussion of these relations is the topic of my paper: The global structure of the set $\{Q(M, K)\}$ of quantizations of the kinematics in a Hilbert space $\mathcal{H}$ is connected with a nonlinear representation of a matrix group $G$ which leads also to a quantization of other observables through nonlinear operators. One obtains e.g. for $M=R^{3}$ a nonlinear representation of the central extension of the inhomogeneous Galilei group $\mathcal{G}(3)$ and furthermore nonlinear Schrödinger equations with given potentials which were also derived in another context in $[1,2]$.

\section{Preview}

We consider scalar nonrelativistic systems localized and moving on a smooth manifold without internal degrees of freedom and external fields (2-forms on $M$ ) and their quantizations (quantum maps) on a suitable Hilbert space $\mathcal{H}$ :

- The set of unitary inequivalent quantum maps $Q$ of systems on $M$ with kinmatic $K(M)$

$$
\left\{Q^{\alpha, D}(M, K)\right\}
$$

is labelled through two quantum numbers $\alpha$ and $D$ which depend on topological and global properties of $M$ and $K(M)[3,4]$, for reviews see [5, 6]. We explain this in section 3 .

- In the case $M=R^{3}$ (with trivial $\alpha$ and a real number $D$ ), presented in section 4 , the set $\left\{Q^{D}\left(R^{3}, K\right)\right\}$ is shown to carry a physically motivated nonlinear representation $\mathbf{N}_{G}$ of a $2 \times 2$ matrix group $G$ which act on a domain $\mathcal{D} \in \mathcal{H}$

$$
\mathbf{N}_{G} \in N: \psi \longrightarrow N[\psi] \text {. }
$$

This implies that unitary inequivalent quantizations in $Q^{D}(\mathfrak{O}), \mathfrak{O}=f, X$, for different $D$ are related through tangent maps with $N$ (see also [4])

$$
\frac{\mathrm{d}}{\mathrm{d} \epsilon}\left(N\left[\psi+i \epsilon Q^{D}(\mathfrak{O}) \psi\right]\right)_{\epsilon=0}=i Q^{D^{\prime}}(\mathfrak{O}) \cdot N[\psi] .
$$

This construction leads also to nonlinear operators from elements of order $\geq 2$ in esa polynomials in $Q^{0}$ and $P^{0}$.

A Lecture on the Occasion of the 70th Birthday of Jiri Niederle 


\section{Borel kinematic $K(M)$ and its quantization}

\section{1 $K(M)$ as global kinematical algebra}

Our systems are localized and moving on a smooth manifold $M$.

To model their localizations we consider a set of regions on $M$ such that this set should contain information on the probabilities to observe the system in a given region. A sufficiently large canonical set of regions is the $\sigma$-algebra $\mathcal{B}(M)$ of Borel sets in $M$. We choose $\mathcal{B}(M)$ as set of position observables.

Smooth motions of systems localized in $B \in \mathcal{B}(M)$ can be described canonically through flows $\phi$ on $M$

$$
B \mapsto B^{\prime}=\phi_{s}^{X}(B)
$$

parameterized with $s$ and characterized through infinitesimal generators $X$ which are contained in the set $V e c t_{0}(M)$ of smooth complete vectorfields in $M$. These covers a large class of motions; their generators $X$ can be chosen as momentum observables. Collecting the results we have:

- The kinematical situation of the system is described with a flow model in $M$. Position observables are modelled with the $\sigma$-algebra $\mathcal{B}(M)$ and momentum observables with vectorfields $V e c t_{0}(M)$. The collection of these observable

$$
K(M)=\left(\mathcal{B}(M), \operatorname{Vect}_{0}(M)\right)
$$

contains through the flow model all possible positions and momenta as global properties of the moving Borel field $\mathcal{B}(M)$. This justifies the name 'Borel' kinematic.

This notion can be generalized $[4,7,8]$ to systems with $k$ internal degrees of freedom and external fields (2-forms on $M)$.

The kinematics $K(M)$ is a time independent quantity. If a time dependence $t$ is introduced to parametrize the motion of the system, e.g. through $t$ dependent states, the quantization $Q$ of $K(M)$ leads to evolution equations which select, with e.g. initial values, the $t$ dependence of the matrix element of the quantized kinematical observable (see e.g. $[1,9,10]$ ).

\subsection{Quantizations of $K(M)$}

We use 'quantization' in the following sense: Consider a set of classical objects, e.g. $K(M)$, a separable Hilbert space $\mathcal{H}$ and the set $S A(\mathcal{H})$ of esa operators (and hence linear) in $\mathcal{H}$. The states of the system are modelled through $\mathcal{H}$. We define 'quantization' as a map $Q$ (quantum map) of this classical object on a cdi domain in $S A(\mathcal{H})$ with certain properties depending on the structure of the system. Hence a quantization of $K(M)$ is a quantum map $Q(M, K)=(\mathrm{Q}, \mathrm{P})$

$$
Q: K(M) \ni(B, X) \mapsto(\mathrm{Q}(B), \mathrm{P}(X) \in S A(\mathcal{H})
$$

on a cdi domain $\mathcal{D}$ in $\mathcal{H}$.

\subsubsection{Quantizations of Borel fields $B(M)$ and Algebraic Properties of $K(M)$}

Following the geometrical background of the flow model and the above notion we interpret the map

$$
\mathrm{Q}: B \mapsto \mathrm{Q}(B) \in S A(\mathcal{H}), B \in \mathcal{B}(M)
$$

through the matrix elements of $\mathrm{Q}(B)$ in a (pure) state with $\psi \in \mathcal{D}$

$$
\left.\mu_{\varphi}(B)=(\psi, \mathrm{Q}(B) \psi)\right) /(\psi, \psi)
$$

as the probability to find the system in this state localized in $B \in \mathcal{B}(M)$. Therefore we assume $\mu_{\psi}: B \longmapsto$ $\mu_{\psi}(B)$ to be a probability measure on $\mathcal{B}(M)$ which implies that $\mathrm{Q}(B)$ is a positive operator valued (pov) measure on $\mathcal{B}(M)$. We assume that the position observable $B$ and also their traumatizations $\mathrm{Q}(B)$ are commutative $^{1}$ and get a projective pov valued measure Q. If $\mathcal{H}$ is realized as $L^{2}(M, \nu)$ ( $\nu$ is a standard measure on $M)$ the $\mathrm{Q}(B)$ act, up to unitary equivalence, as a multiplication operator with the characteristic function of $B$

$$
\mathrm{Q}(B) \psi=\chi(B) \psi
$$

Finally we induce a quantum map $\mathrm{Q}$ of the set $C^{\infty}(M)$ of real smooth functions $f(m)$ on $m \in M$ via the spectral theorem

$$
\mathrm{Q}: C^{\infty}(M) \ni f \mapsto \mathrm{Q}\left(f(m)=f(m) \in S A\left(L^{2}(M, \nu)\right)\right.
$$

The model for position observables with smooth functions $\left.f \in C^{\infty}(M)\right)$ instead with Borel sets $B \in \mathcal{B}(M)$ is motivated because one can equivalently write $K(M)$ as

$$
K(M)=\left(C^{\infty}(M), \operatorname{Vect}_{0}(M)\right)
$$

which implies an algebraic structure of $K(M)$ : the abelian Lie-algebra $C^{\infty}(M)$ and the algebra of smooth vectorfields $V \operatorname{ect}_{0}(M)$ which act together on $M$ as semidirect sum

$$
K(M)=C^{\infty}(M) \oplus_{s} V e c t_{0}(M) .
$$

Hence $K(M)$ can be viewed as an ( $\infty$ dim) global infinite dimensional Lie-algebraic symmetry of a system on $M$. It can also be considered as Lie-algebra of an inhomogeneous subgroup of the diffeomorphism group $\operatorname{Dif} f(M)$ which was used in [11].

\footnotetext{
${ }^{1}$ Certain types of noncommutative positions can also be discussed in this approach.
} 
This symmetry (10) reflects the physics in the flow model and it is plausible to assume that the symmetry survives the quantization map Q and leads after quantization in $L^{2}(M, \nu)$ to a partial realization of $K(M)$ in (10). We add 'partial' because two esa elements in $K(M)$ on $\mathcal{D}$ belong to $\mathrm{Q}(M, K)$ only if their linear combinations and commutators are also esa on $\mathcal{D}$. Collecting the result we have:

- Quantum maps Q of position observable $f \in$ $C^{\infty}(M)$ lead to $\mathrm{Q}(f)$ which act as multiplication operators in $L^{2}(M, \nu)$. This allows to view $K(M)$ as global infinite dimensional Lie-algebraic symmetry of the system which is assumed to survive the quantum map.

\subsubsection{Quantizations of $V \operatorname{ect}_{0}(M)$}

Momentum observables in $K(M)$ are introduced as flow generators $X \in V$ ect $t_{0}(M)$ of $\phi^{X}$. Following our above arguments their quantum map $\mathrm{P}$ should again be based on the geometrical roots and must respect the consistency with $\mathrm{Q}(f)$ in $(10)$.

\section{I.}

The observable $X$ act in our flow model as differential operators on position observables in $C^{\infty}(M)$. Hence it is plausible to assume that also $\mathrm{P}(X) \in S A\left(L^{2}().\right)$ is a finite order differential operator on $L^{2}(M, \nu)$. However, a realization of this assumption is difficult. This is because a definition of differential operators in $L^{2}(M, \nu)$ needs a notion of the differentiation of complex functions $\psi(m)$ on a smooth manifold $M$ which are square integrable in respect to the measure $\nu$. This measure theoretic property contains no information on their differentiability. Hence additional assumptions are necessary to realize momentum operators $\mathrm{P}(X)$ as PDO's.

To formulate such assumptions we note that differentiation on a smooth manifold $M$ is given through its definition, and differentiation on the complex plane $C$ is a standard notion. This implies technically the existence of differential structures $\mathrm{Ð}(M)$ on $M$ and $\mathrm{Ð}(C)$ on $C$. For the differentiation of complex functions on $M$ in $L^{2}(M, \nu)$ we need technically a differential structure $\mathrm{Ð}(M \times C)$ on the point set $M \times C$ with the restrictions

$$
Ð(M \times C) / M=Ð(M), \quad Ð(M \times C) /{ }_{C}=\nsupseteq(C) .
$$

Without going into mathematical details, including the definition of $Ð$ and interesting applications, we need for $\mathrm{P}$ some results on differential structures with (11). A possibility is to look on complex line bundles $\eta$ over $M$ with hermitean metric $<,>$. This is because there exists with $\eta$ an isometrically isomorphic complex line bundle $\eta_{0}$ with hermitean metric $<,>_{0}$ and a differentiable structure $Đ(M \times C)$ with (11). Hence we are interested in complex line bundles with metric $<,>$ with Hermitean connection $\nabla$ and in their clas- sification up to equivalence. This is well known, we refer e.g. to $[12,13]$. Because we are dealing with a system without external fields the connection is flat. The inequivalent line bundles with flat connection are labelled by the character group $\pi_{1}^{*}(M)$ of the fundamental group of $M$.

We have to relate the sections $\sigma$ of a line bundle $\eta$ with the elements $\psi$ of $L^{2}(M, \nu)$. The sections $\sigma$ in $\eta$ form a complex vectorspace and, together with $\nu$ and $<,>$, the 'square' integrable ones form a Hilbert space $L^{2}(\eta,<,>, \nu)$. A dense domain of smooth sections in $\eta$ can be embedded in a dense domain $\mathcal{D}$ in $L^{2}(M, \nu)$. Hence we use this domain $\mathcal{D}$ to define differential operators.

In general there are a large number of nonequivalent differentiable structures on $M \times C$ which lead to different dense domains in $L^{2}(M, \nu)$. Hence one can view the definition of differential operators with a domain problem.

\section{II.}

In part I., we explained how to introduce a differential structure. Now we refer again to the geometrical roots to argue that our differential operators are of finite order. Classical generators $X$ of with support $\operatorname{supp}(X)$ moves with $\phi^{X}$ the characteristic function $\chi(B)$ resp. the support of $f$. After quantization the situation should be analogous. This motivates a locality condition for $\mathrm{P}(X)$

$$
\operatorname{supp}(\mathrm{P}(X) \psi) \subseteq \operatorname{supp}(\psi), \psi \in L^{2}(M, \nu) .
$$

With Peetre's theorem [14] this locality condition and a differentiable structure $\mathrm{Ð}(M \times C)$ yields for $\mathrm{P}(X)$ differential operators of finite order.

Collecting the results we have:

- The quantum map P of momentum observable $X$ leads to differential operators of finite order on a domain in $L^{2}(M, \nu)$ which is embedded on a domain in $L^{2}(\eta,<,>, \nu)$ spanned by square integrable sections of a complex line bundle $\eta$ on $M$ with a Hermitean metric and a flat Hermitean connection.

\subsubsection{Quantizations of the Borel kinematic $K(M)$}

With the properties for $\mathrm{Q}(f)$ and $\mathrm{P}(X)$ at the end of section 3.2.1 and 3.2.2 we construct the quantum map $\mathrm{Q}(M, K)$. The result reflects the classification of flat complex line bundles with a Hermitean metric and a Hermitean connection. A further classifying real number $D$ is related to the global Lie-algebraic symmetry $K(M)(10)$ and the consistency of the partial realization in $\mathrm{Q}(M, K)$.

\section{Classification Theorem for Quantum Borel kine- matics \\ Inequivalent irreducible quantum maps $Q(M, K)$ from $K(M)$ to a cdi domain $\mathcal{D}_{Q}$ in $S A\left(L^{2}(M, \nu)\right)$}




$$
Q^{\alpha, D}(M, K)=\left(\mathrm{Q}^{\alpha, D}, \mathrm{P}^{\alpha, D}\right.
$$

are labelled by two numbers $\alpha, D$

$$
\alpha \in \pi_{1}^{*}(M), D \in \mathbb{R} .
$$

The domain $\mathcal{D}_{Q} \subset L^{2}(M, \nu)$ is obtained through an embedding in the Hilbert space $L^{2}(\eta,<,>, \nu)$ spanned through square integrable sections of the complex line bundle $\eta$ on $M$ with Hermitean metric and a flat Hermitean connection.

The $\alpha, D$ are quantum numbers in the sense of Wigner. Quantizations $\mathrm{P}^{\alpha, D}$ for different $\alpha$ and/or $D$ are unitary inequivalent. Quantizations $\mathrm{Q}^{\alpha, D}$ which act on $\mathcal{D}$ as

$$
\left.\mathrm{Q}^{\alpha, D}(f(m))\right)=f(m), \quad f(m) \in C^{\infty}(M, R)
$$

are independent of $\alpha$ and $D$. For $\mathrm{P}^{\alpha, D}$ on $\mathcal{D}$ one obtains a first order PDO through Lie-derivatives and as the zero order part a smooth section of the endomorphism bundle which depends on $D$ and $\alpha$ (see the example in section 3.2.4 and [3]).

There are many applications. We refer to the list in [4] and e.g. to quantizations on a trefoil manifold [15] and on two and higher dimensional configuration manifolds for $n$ identical particles [16] including anyons.

Physical effects of quantum maps $Q^{\alpha, D}$ with $D=0$ through quantum numbers $\alpha$ are experimentally observed. However, for $Q^{\alpha, D}$ with $D \neq 0$ our derivation contains no information on the interpretation and hints for its physical relevance; even in the case for trivial $\alpha$ the physical meaning of $D$ is unknown. Furthermore $Q^{\alpha, D}$ with $D \neq 0$ implies as a quantum map of the kinematics no information on the time dependence of the system, i.e. of its dynamics and furthermore no rule for the quantization (up to orderings) of higher order $(\geq 2)$ polynomials of momentum and position observable.

A possible answer to these questions was proposed by Jerry Goldin and HDD [1]. They introduced for $M=R^{3}$ a time dependence of $\psi$ through particle conservation and obtained with $Q^{\alpha, D}\left(R^{3}, K\right)$ after 'gauge generalisation' nonlinear Schrödinger equations with nonlinear terms proportional to $D$. The procedure can be generalized to systems on $M$. These results indicate a hidden nonlinear structure of $\left\{Q^{\alpha, D}\left(M, K^{3}\right\}\right.$ which will be explored in section 4 .

\subsubsection{Quantizations on $M=R^{3}$}

As an example for the classification theorem we consider $M=R^{3}$ with trivial $\pi_{1}^{*}\left(R^{3}\right)$ and $X=\vec{g}(x) \vec{\nabla}$, $f=f(x)$. We find for $Q^{D}\left(R^{3}, K\right)$ in $\mathcal{D} \in L^{2}\left(R^{3}, \mathrm{~d} x^{3}\right)$

$$
\begin{aligned}
\mathrm{Q}^{D}(f) & =f(x), \\
\mathrm{P}^{D}(X) & =\frac{1}{i} \vec{g}(x) \vec{\nabla}+\left(\frac{1}{2 i}+D\right) \operatorname{div} \vec{g}(x) .
\end{aligned}
$$

$Q^{D=0} \equiv Q^{0}$ is the canonical quantization. The maps $Q^{D}$ and $Q^{D^{\prime}}$ are unitarily inequivalent for $D \neq D^{\prime}$. The expectation values can be scaled according to their physical interpretation with automorphisms of $\operatorname{Vect}_{0}(M) \ni X \longmapsto a X$ and $C^{\infty}(M, R) \ni f \longmapsto b f$.

\section{A nonlinear symmetry of $\left\{\mathrm{Q}^{D}\right\}$}

\subsection{Nonlinear transformations and operators}

We use the above set $\left\{Q^{D}\left(R^{3}, K\right)\right\}$ to look for a 'hidden non linear structure'. Because nonlinearities are often a result of nonlinear transformations it is plausible to try physically motivated nonlinear transformations $N$ of $\psi \in L^{2}\left(R^{3}, d^{3} x\right)$

$$
N: \psi \longrightarrow N[\psi]=N(\psi) \psi
$$

We assume that $N$ act as multiplication operators and depend only on $\psi$, i.e. not on derivatives of $\psi$ and not explicitly on $x, t$ (domain and range questions are not discussed in this paper). These transformation $N[\psi]$ could imply singularities e.g. in evolution equations, which are not important here. In the case for multivalued $N[\psi]$ one has to show that relevant results are unique and independent from the choice of the representatives of the ray $\left\{\psi_{\tau} \mid \psi \exp i \tau, \tau\right.$ real $\}$ which describe physical equivalent states.

To construct from esa operators $A$ through $N$ operators $A_{N}$, which may be linear or nonlinear, we again use the flow model. The flow with generator $X$ corresponds after quantization to a strongly continuous one parameter unitary group $U_{\epsilon}$ with an esa generator. This generator appears through a tangent $\operatorname{map} \frac{\mathrm{d}}{\mathrm{d} \epsilon}\left(U_{\epsilon} \psi\right)_{\epsilon=0}$ on a path $\left\{U_{\epsilon} \psi, \in[-1,+1]\right\}$ in $L^{2}\left(R^{3}, \mathrm{~d}^{3} x\right)$. With this in mind we construct $A_{N}$ from a given esa $A$ with $V_{\epsilon}=\exp i \epsilon A$ via a tangent map $N\left[V_{\epsilon} \psi\right]$ with $N$ on a corresponding nonlinear path

$$
\begin{aligned}
\frac{\mathrm{d}}{\mathrm{d} \epsilon}\left(N\left[V_{\epsilon} \psi\right]\right)_{\epsilon=0}= & \frac{\mathrm{d}}{\mathrm{d} \epsilon}(N[\psi+i \epsilon A \psi])_{\epsilon=0} \equiv \\
& i A_{N} \cdot N[\psi] .
\end{aligned}
$$

on a domain in which the limit exists. For differential operators $A_{N}$ this domain can be extended in $L^{2}\left(R^{3}, \mathrm{~d}^{3} x\right)$. For some not esa linear $A$ this construction is also possible.

\subsection{Choice of nonlinear transformations}

Our aim is to determine a set $\mathbf{N}$ of nonlinear transformations such that $\mathrm{Q}^{D}, \mathrm{P}^{D}$ in (15)

$$
\mathrm{Q}^{D}=f, \quad \mathrm{P}^{D}=\frac{1}{i} \vec{g} \cdot \vec{\nabla}+\left(\frac{1}{2 i}+D\right) d i v \vec{g}
$$


are connected for different $D$ through a tangent map with $N \in \mathbf{N}$

$$
Q^{D^{\prime}}=Q_{N}^{D} \text { with } \mathrm{Q}^{D^{\prime}}=\mathrm{Q}_{N}^{D}, \quad \mathrm{P}^{D^{\prime}}=\mathrm{P}_{N}^{D}
$$

An evaluation of these conditions is indeed possible; the calculation is straightforward. With a (non unique) polar decomposition $\psi=R \exp i S$ we find for the nonlinear transformations $N$

$$
N(\gamma, \Lambda) \psi=\exp i(\gamma \ln R+(\Lambda-1) S) \cdot \psi .
$$

They build a nonlinear representation $\mathbf{N}_{G}=$ $\{N(\gamma, \Lambda)\}$ of two parameter $(\gamma, \Lambda)$ group $G$. The group $\mathbf{N}_{G}$ was derived in a different context and with other assumptions in [17]. For the corresponding tangent maps we find

$$
\begin{gathered}
\mathrm{Q}^{D}=\mathrm{Q}_{N(\gamma, \Lambda)}^{0} \text { with } \gamma=2 D, \text { M.arbitrary } \\
\mathrm{Q}^{D^{\prime}}=\mathrm{Q}_{N(\gamma, \Lambda)}^{D} \text { with } \gamma=2\left(D^{\prime}-D\right), \quad \Lambda=1 .
\end{gathered}
$$

Hence our search for a hidden symmetry was successful. The result implies:

- Quantizations $Q^{D}$ and $Q^{D^{\prime}}$ are inequivalent in respect to the group of unitary transformations but 'equivalent' in respect to a nonlinear realization $\mathbf{N}_{G}$ of a matrix group $G$ with one and for $D=0$ and for $D^{\prime} \neq 0$ with two real parameters. The quantum number $D$ leads to a nonlinear representation of a $G$ symmetry of $\left\{Q^{D}\left(K\left(R^{3}\right)\right)\right\}$.

\subsection{Nonlinear quantizations and symmetries}

We now extend our construction from linear first and zero order differential operators in $\mathrm{Q}\left(R^{3}, K\right)$ to esa higher order differential operators operators in polynomials of canonically quantized observables

$$
\mathbf{D} \in \mathcal{P}_{\text {esa }}\left(\mathrm{Q}^{0}(f), \mathrm{P}^{0}(X)\right) .
$$

An application of tangent maps leads to

$$
N(\gamma, \Lambda): \quad \mathbf{D} \mapsto \mathbf{D}_{N(\gamma, \Lambda)}
$$

i.e. to a two parameter set of nonlinear differential operators $\mathbf{D}_{N(\gamma, \Lambda)}$. This nonlinear 'extension' of canonically quantized polynomials $\mathbf{D}$ of classical observables through tangent maps with $\mathbf{N}_{G}$ is an attempt for a formal path to nonlinear 'extensions' of quantum mechanics. However, an interpretation of nonlinear operators and nonlinear evolutions as in (linear) quantum theory is not possible. Results from a nonlinear theory can be interpreted in some approximation as in the linear case, e.g. the eigenvalues of a nonlinear Schrödinger equation (22) (see [18]). A complete mathematical framework and convincing physical interpretations for a nonlinear 'extension' is not known.

With (21) one obtains an already mentioned nonlinear realization of $\mathcal{G}(3)$ with linear generators of the
Galilei-algebra with the exception of the free Hamiltonian which appears as nonlinear operator. Furthermore one gets with $N \in \mathbf{N}_{G}$ from a given linear Schrödinger equation a nonlinear one with nonlinear part $F[\psi]$ which depends on $\gamma, \Lambda$. This nonlinear equation was generalized in [19] to a nonlinearisable Schrödinger equation (known as the Doebner Goldin equation) with real coefficients $D, \lambda_{1}, \ldots, \lambda_{5}$

$$
\begin{aligned}
& i \hbar \partial t=\left(-\hbar^{2} / 2 m \triangle+F[\psi]\right) \psi \\
& F[\psi]=\frac{i \hbar D}{2} \frac{\triangle \rho}{\rho}+\hbar D\left\{\lambda_{1} R_{1}+\ldots+\lambda_{5} R_{5}\right\}
\end{aligned}
$$

with

$$
\begin{aligned}
\rho & =\bar{\psi} \psi, \quad J=\frac{\hbar}{2 m i}\{\bar{\psi} \nabla \psi-\nabla \psi \bar{\psi}\} \\
R_{1}[\psi] & =\frac{\nabla \cdot J}{\rho}, R_{2}[\psi]=\frac{\triangle \rho}{\rho}, R_{3}[\psi]=\frac{J^{2}}{\rho^{2}} \\
R_{4}[\psi] & =\frac{J \cdot \nabla \rho}{\rho^{2}}, R_{5}[\psi]=\frac{\nabla \rho \cdot \nabla \rho}{\rho^{2}}
\end{aligned}
$$

\section{Conclusion}

We announced in the preview that we would relate global and nonlinear structures of quantum maps $\mathrm{Q}(M, K)$ for the Borel kinematics $K(M)$ of nonrelativistic systems on $M$ without internal degrees of freedom and external fields with application for $\mathrm{Q}\left(R^{3}, K\right)$.

- Inequivalent quantum maps $\left\{Q^{\alpha, D}(M, K)\right\}$, labelled through quantum numbers $\alpha, D$ in the sense of Wigner which reflect topological and global properties of $M$ and $K(M)$. Experiments to measure effects in $Q^{\alpha, D}$ are known for $D=0$.

- For $M=R^{3}$ and $D \neq 0$ these global structures imply that $\left\{Q^{D}\left(R^{3}, K\right)\right\}$ carries a nonlinear representations $\mathbf{N}_{G}$ of a $2 \times 2$ matrix group $G$.

- For esa differential operators $\mathbf{D}$ of order $\geq 2$ in the polynomial set $\mathcal{P}_{\text {esa }}\left(\mathrm{Q}^{0}(f), \mathrm{P}^{0}(X)\right)$ one obtains nonlinear differential operators $\mathbf{D}_{N}$ through tangent maps with $N \in \mathbf{N}_{G}$. Nonlinear versions of symmetry and dynamical symmetry algebras are available. Our construction may be viewed as part of a path to a nonlinear extension of quantum mechanics. This may be relevant in the case that precision experiments show a nonlinear character and corresponding nonlinear evolutions based on a global character of $M$ and $K(M)$.

\section{References}

[1] Doebner, H.-D., Goldin, G. A.: Phys. Lett. A 162, 397 (1992).

[2] Doebner, H.-D., Goldin, G. A.: Phys. Rev. A 54, 3764 (1996).

[3] Angermann, B., Doebner, H.-D., Tolar, J.: Lecture Notes in Math. Vol. 1037 (1983). 
[4] Doebner, H.-D., Tolar, J.: in Symmetry in Science XIV Kluver, Dordrecht (2004).

[5] Doebner, H.-D., Stovicek, P., Tolar, J.: Rev. Math. Phys. 13, 799 (2001).

[6] Ali, S., English, M.: Rev. Math. Phys. 17, 205 (2005).

[7] Drees, M.: Zur Kinematik lokalisierbarer quantenmechanischer Systeme unter Berücksichtigung innnerer Freitsgrade und äusserer Felder, PhD. thesis TU Clausthal (1992).

[8] Nattermann, P.: Dynamics in Borel Quantisation: Nonlinear Schrödinger Equations vs. Master Equations, PhD. thesis TU Clausthal (1997).

[9] Hennig, J. D.: in Nonlinear, Deformed and Irreversible Quantum Systems, World Scientific (1995).

[10] Doebner, H.-D., Hennig, J. D.: in Symmetry in Science IX, Plenum Press (1996).

[11] Goldin, G. A., Menikoff, R., Sharp, D. H.: J. Math. Phys. 21, 650 (1980).
[12] Kostant, B.: in Quantisations and unitary representations, Lecture Notes in Math. Vol. 170 (1970).

[13] Weil, A.: Variete Kähleriennses, Herman, Paris (1958).

[14] Kahn, W.: Introduction to Global Analysis, Academic Press, London (1980).

[15] Doebner, H.-D., Groth, W.: J. Phys. A 30, L503 (1997).

[16] Doebner, H.-D., Groth, W., Hennig, J. D.: J. Geom. Phys. 31, 35 (1998).

[17] Doebner, H.-D., Goldin, G. A., Nattermann, P.: J. Math. Phys. 40, 49 (1996).

[18] Doebner, H.-D., Manko, V. I., Scherer, W.: Phys. Lett. A 268, 17 (2000).

[19] Doebner, H.-D., Goldin, G. A.: J. Phys. A Math. Gen. 27, 1771 (1994).

H.-D. Doebner

E-mail: asi@pt.tu-clausthal.de, doebner@t-online.de

Technical University of Clausthal

Institute for Energy Research and Physical Tecnology 